PAPER

\section{Broadband mode in proton-precession magnetometers with signal processing regression methods}

To cite this article: Alexey Y Denisov et al 2014 Meas. Sci. Technol. 25055103

View the article online for updates and enhancements.
Related content

The low cost Proton Precession Magnetometer developed at the Indian Institute of Geomagnetism

P. Mahavarkar, S. Singh, S. Labde et al.

An on-line digital phase sensitive detector in the range $2 \mathrm{mHz}-2 \mathrm{kHz}$

G J Gerritsma, W J van Weezep, J A

Overweg et al.

Research on a secondary tuning algorithm based on SVD \& STFT for FID signal Huan Liu, Haobin Dong, Jian Ge et al

\section{Recent citations}

Systematic and statistical uncertainties of the hilbert-transform based high-precision FID frequency extraction method Ran Hong et al

An optimized free induction decay signal sensing coil and its matching circuit for miniaturized Overhauser geomagnetic sensor

Hongpeng Wang et al

- Gradient spin echo enhanced proton precession maqnetometer: A novel system for field gradient measurement Farrokh Sarreshtedari et al

\title{
Intensive Short Courses
}

Sun, Oct 10 \& Mon, Oct 11

Providing students and professionals with in-depth education on a wide range of topics 


\title{
Broadband mode in proton-precession magnetometers with signal processing regression methods
}

\author{
Alexey Y Denisov ${ }^{1}$, Vladimir A Sapunov ${ }^{1}$ and Boris Rubinstein ${ }^{2}$ \\ ${ }^{1}$ Quantum Magnetometry Laboratory, Ural Federal University after First President of Russia \\ B N Yeltsin, Mira St., 19, Yekaterinburg 620002, Russia \\ ${ }^{2}$ Stowers Institute for Medical Research, 1000 E 50th St, Kansas City, MO 64110, USA \\ E-mail: alex@dpt.ustu.ru
}

Received 6 September 2013, revised 30 December 2013

Accepted for publication 10 January 2014

Published 27 March 2014

\begin{abstract}
The choice of the signal processing method may improve characteristics of the measuring device. We consider the measurement error of signal processing regression methods for a quasi-harmonic signal generated in a frequency selective device. The results are applied to analyze the difference between the simple period meter processing and regression algorithms using measurement cycle signal data in proton-precession magnetometers. Dependences of the measurement error on the sensor quality factor and frequency of nuclear precession are obtained. It is shown that regression methods considerably widen the registration bandwidth and relax the requirements on the magnetometer hardware, and thus affect the optimization criteria of the registration system.
\end{abstract}

Keywords: signal processing, magnetic resonance, regression algorithm, magnetometer, zero-crossing problems

\section{Introduction}

The development of the processing algorithm for nuclear precession signals starts with a simple period meter method used in many investigations devoted to the analysis and optimization of proton magnetometers. The choice and suitability of this specific algorithm usually are not discussed and only signal-to-noise parameters of the device are analyzed [1-3]. Choice of the specific algorithm for the device signal processing can result in a number of effects and requirements on accuracy and operation speed; it also generates specific dependences of the measurement error on magnetometer parameters $[4,5]$. In this paper, we demonstrate the fundamental difference of the period meter method from regression algorithms accounting for measurement cycle signal data. The most important differences concern, first, changes in the optimization criteria of the registration system, and, second, relaxation of the requirements on the system frequency response.

\section{Digital signal processing}

Consider key algorithms determining absolute value of the measured magnetic field induction $B_{0}$ related to the signal period $T^{0}$ of the nuclear moments free precession in a given magnetic field:

$$
B_{0}=\gamma_{\mathrm{p}}^{-1} \omega_{0}=2 \pi\left(\gamma_{\mathrm{p}} T^{0}\right)^{-1},
$$

where $\gamma_{\mathrm{p}}=2 \pi \cdot 0.0425764 \mathrm{rad}(\mathrm{nT} \mathrm{s})^{-1}$ [6] denotes the proton-gyromagnetic ratio and $\omega_{0}$ is the signal frequency. The simplest implementation, the method of period meter (MPM), computes the average period, $\bar{T}^{\mathrm{pm}}[7]$,

$$
\bar{T}^{\mathrm{pm}}=\frac{2}{n N}\left(t_{N}-t_{0}\right)
$$

where $N$ is the total number of signal-recorded zero crossings during the measurement, $n$ is the number of precession half-periods (digitization step) between the two subsequent recorded crossings (each $n$th half-period is registered), $t_{i}$ denotes the $i$ th zero crossing time and further on for simplicity we set $t_{0}=0$. This technique was widely used in magnetometry, and we use it as the basis for the analysis 
of a more precise algorithm of regression type-the leastsquares method (LSM) [8-10] that finds the average period $\bar{T}^{\text {ls }}$ as follows:

$$
\bar{T}^{\mathrm{ls}}=\frac{12}{n N(N+1)(N+2)} \sum_{i=0}^{N}(2 \mathrm{i}-N) t_{i} .
$$

The main advantage of this algorithm compared to MPM (2) is that it uses the additional data obtained within the measurement cycle.

\section{Signal processing algorithm based on arbitrary linear regression}

Consider an ideal linear frequency selective system of registration with constant parameters and limited size bandwidth centered at $\omega_{r}$, which generates an output signal for further processing. The model of signal processing assumes that the system input signal is the sum of a quasi-harmonic $A(t) \cos \left(\omega_{0} t+\varphi\right)$ and input noise component $u(t)$. We assume that the input noise is the normal stationary random wide-band process with known spectral intensity $W(\omega)$. With the assumption of a slow varying envelope $A(t)$, we use a stationary signal approximation and, neglecting the contribution of the system transition processes, write the detector output signal

$F(t)=S(t) \cos \left(\omega_{0} t+\phi\right)+U(t), \quad S(t)=A(t) K\left(\omega_{0}\right)$,

where $K\left(\omega_{0}\right)$ denotes the system transfer function absolute value and $U(t)$ is the output noise characterized by the dispersion

$$
\sigma^{2} \equiv\left\langle U^{2}(t)\right\rangle=\frac{1}{2 \pi} \int_{-\infty}^{\infty} K(\omega)^{2} W(\omega) \mathrm{d} \omega,
$$

and by the normalized correlation function

$$
\begin{aligned}
\rho(\tau) & \equiv \sigma^{-2}\langle U(t+\tau) U(t)\rangle \\
& =\frac{1}{2 \pi \sigma^{2}} \int_{-\infty}^{\infty} K(\omega)^{2} W(\omega) \cos \omega t \mathrm{~d} \omega .
\end{aligned}
$$

We assume that the comparator at the system output registers zero crossings $t_{i}$, and then, using a linear regression method we compute some quantity $P$ :

$$
P=\sum_{i=0}^{N} a_{i} t_{i}
$$

where $a_{i}$ are the weights of $t_{i}$. The measurement error of $P$ is determined by the noise level, the transition process in the registration system and the comparator non-zero level $\Lambda \neq 0$ that defines the time moments $t_{i}$. These factors lead to the non-zero shift of the actual values $t_{i}$ compared to the values $t_{i}^{0}=i n T^{0} / 2$, corresponding to the non-decaying signal without a noise component. Assuming the condition of the comparator level $\Lambda=F\left(t_{i}\right)$ and using an approximation $S_{i} \equiv S\left(t_{i}^{0}\right) \approx S\left(t_{i}\right)$ we obtain the delay for the $i$ th zero crossing

$$
\begin{aligned}
\Delta t_{i} & \equiv t_{i}-t_{i}^{0}=\operatorname{sign}\left[\sin \left(\omega_{0} t_{0}^{0}+\phi\right)\right] \frac{(-1)^{i n}}{\omega_{0}} \\
& \times\left[\arcsin \frac{\Lambda}{S_{0}}-\arcsin \frac{\Lambda-U\left(t_{i}\right)}{S_{i}}\right] .
\end{aligned}
$$

Using the last relation and the output noise characteristics (5) and (6), we compute the statistical moments of the random variable $\Delta t$. Assuming $\Lambda \ll S_{i}$ and retaining the leading terms only in the expansion in the noise/signal ratio, we arrive at the following expressions for the mean and covariance:

$$
\left\langle\Delta t_{i}\right\rangle \approx \operatorname{sign}\left[\sin \left(\omega_{0} t_{0}^{0}+\phi\right)\right] \frac{(-1)^{i n}}{\omega_{0}} \frac{\Lambda}{S_{i}}\left(1-\frac{S_{i}}{S_{0}}+\frac{\sigma^{2}}{2 S_{i}^{2}}\right),
$$

$$
\begin{aligned}
& \left\langle\Delta t_{i} \Delta t_{j}\right\rangle-\left\langle\Delta t_{i}\right\rangle\left\langle\Delta t_{j}\right\rangle \approx \frac{(-1)^{n(i-j)}}{\omega_{0}^{2}} \\
& \times \frac{\sigma^{2}}{\sqrt{\left(S_{i}^{2}-\Lambda^{2}\right)\left(S_{j}^{2}-\Lambda^{2}\right)}} \rho\left(\left|t_{i}^{0}-t_{j}^{0}\right|\right) .
\end{aligned}
$$

Using these results one can find the absolute and random measurement error for various types of processing of zero crossing times. Consider the random measurement error. By (9), the dispersion of $P$ reads

$$
\begin{aligned}
\sigma_{P}^{2} & =\sum_{i=0}^{N} \sum_{j=0}^{N} a_{i} a_{j}\left(\left\langle\Delta t_{i} \Delta t_{j}\right\rangle-\left\langle\Delta t_{i}\right\rangle\left\langle\Delta t_{j}\right\rangle x\right) \\
& \approx \frac{\sigma^{2}}{\omega_{0}^{2}} \sum_{i=0}^{N} \sum_{j=0}^{N} \frac{(-1)^{n(i-j)} a_{i} a_{j}}{\sqrt{\left(S_{i}^{2}-\Lambda^{2}\right)\left(S_{j}^{2}-\Lambda^{2}\right)}} \rho\left(\left|t_{i}^{0}-t_{j}^{0}\right|\right) .
\end{aligned}
$$

Consider the case when the decay time of the correlation function $\rho$ (correlation time $\tau_{c}$ ) is much less than the signal measurement time. Also, assume that the decay rate of the function $\rho(t)$ is much faster than the rate of change of the coefficient $a\left(t_{i}\right) /\left(S^{2}\left(t_{i}\right)-\Lambda^{2}\right)^{1 / 2}$. These approximations allow us to consider the correlation function as $\delta$-function compared to the abovementioned coefficient and we obtain the final expression for the dispersion of the quantity $P$ as

$$
\begin{aligned}
\sigma_{P}^{2} & \approx \frac{\sigma^{2}}{\omega_{0}^{2}} \sum_{i=0}^{N} \frac{a_{i}^{2}}{S_{i}^{2}-\Lambda^{2}} \sum_{j=0}^{N}(-1)^{n(i-j)} \rho\left(\left|t_{i}^{0}-t_{j}^{0}\right|\right) \\
& \approx\left(\frac{\sigma f_{c}}{S_{0} \omega_{0}}\right)^{2} \sum_{i=0}^{N} \frac{S_{0}^{2} a_{i}^{2}}{S_{i}^{2}-\Lambda^{2}},
\end{aligned}
$$

where the correlation factor $f_{c}$ reads

$$
f_{c}^{2}=\sum_{k=-\infty}^{\infty}(-1)^{k n} \rho\left(|k| n T^{0} / 2\right) \text {. }
$$

Using (6), the relation

$$
\sum_{k=-\infty}^{\infty} \exp (2 \pi \mathrm{i} x k)=\sum_{m=-\infty}^{\infty} \delta(x-m)
$$

and symmetry of the functions $K$ and $W$ with respect to $\omega$, we obtain

$$
f_{c}^{2}=\frac{2 \omega_{0}}{\pi n \sigma^{2}} \sum_{m=-[n / 2]}^{\infty} K\left(\omega_{0}(1+2 m / n)\right)^{2} W\left(\omega_{0}(1+2 m / n)\right),
$$

where $[\cdots]$ denotes the integer part. In the case of a narrow bandwidth system, we have from (11)

$$
\sigma_{P}^{2} \approx \frac{2 W\left(\omega_{r}\right)}{t_{N} A_{0}^{2}} G(\lambda+1)^{2}, \quad G=\frac{N}{\omega_{0}^{2}} \sum_{i=0}^{N} \frac{S_{0}^{2} a_{i}^{2}}{S_{i}^{2}-\Lambda^{2}},
$$


where the correction $\lambda$ is defined by the relation $(\lambda \rightarrow 0$ at $\omega_{0}=\omega_{r}$ for a negligibly small bandwidth)

$(\lambda+1)^{2}=\sum_{m=-[n / 2]}^{\infty} \frac{K\left(\omega_{0}(1+2 m / n)\right)^{2}}{K\left(\omega_{0}\right)^{2}} \frac{W\left(\omega_{0}(1+2 m / n)\right)}{W\left(\omega_{r}\right)}$.

Thus, almost all the dependence of the error on the frequency response is accumulated in $\lambda$. Consider the case of a signal close to $\omega_{r}$ and broadband noise; when $\tau_{c}>n T^{0} / 2$, we find that $2 \omega_{0} / n$ is larger than the bandwidth, and we can neglect $\lambda$ compared to the unity, i.e., neglect the frequency response contribution to the measurement error. This means that the frequency dependence is contributed by the factor $G$ in (13), i.e., by the algorithm and the signal envelope, and does not depend on the registration system bandwidth.

\section{Proton magnetometer model}

A registration system of a proton-precession magnetometer includes a working substance, a receiving-polarizing coil of the sensor, a tuning capacitor, an electronic commutator switching the coil between the polarization and measurement regimes, a low-noise broadband linear amplifier and an analogue data digitizer. We assume that the generator frequency is high enough to neglect the quantum noise effect during the digitization process. The leading source of the error is the thermal noise of the receiving coil, whose spectral properties are determined by sensor electrical parameters.

The electric circuit of the proton sensor is an oscillatory circuit consisting of inductance $L$, measuring coil resistance $R$ and tuning capacity $C$. The absolute value of the circuit transfer function reads

$$
K=\frac{Q}{\sqrt{\alpha^{2}+Q^{2}\left(\alpha^{2}-1\right)^{2}}},
$$

where $\alpha=\omega_{0} / \omega_{r}$ denotes the ratio of the precession frequency $\omega_{0}$ to the resonance frequency $\omega_{r}=(L C)^{-1 / 2}$ and $Q=\tau_{c} \omega_{r} / 2$ is a quality factor (here $\tau_{c}=2 L / R$ denotes the circuit correlation time). The circuit input is presented as a sum of working substance signal $f(t)=A_{0} \exp \left(-t / T_{2}\right) \cos \left(\omega_{0} t+\phi\right)$ and noise $u(t)$ components; here $A_{0}$ is the initial amplitude, normalized to the circuit input, and $T_{2}$ is the transversal relaxation time.

Assuming that the coil thermal fluctuations are the only source of noise in the system, we find the spectral intensity at temperature $T$ using the Nyquist theorem [11] as $W(\omega)=2 k_{B} T R$, where $k_{B}$ denotes the Boltzmann constant. This assumption leads to the following characteristics of the circuit output noise (see (5), (6)):

$$
\sigma^{2}=\omega_{r} Q W\left(\omega_{r}\right) / 2=\omega_{r} Q k_{B} T R,
$$

and

$$
\rho(t)=\mathrm{e}^{-t / \tau}\left(\cos \tilde{\omega}_{r} t+\frac{\sin \tilde{\omega}_{r} t}{\tilde{\omega}_{r} \tau_{c}}\right),
$$

where $\tilde{\omega}_{r}=\omega_{r} \sqrt{1-(2 Q)^{-2}}$ is the natural frequency of the circuit. The analysis of this model of the proton magnetometer using the processing algorithms mentioned above was given in [4].

\section{Magnetometer measurement error}

Using the relation (1) and the results (10) and (16) we find the expression for the mean standard deviation (MSD) of the magnetic induction modulus $\sigma_{B}^{2} \equiv\left\langle(B-\langle B\rangle)^{2}\right\rangle=$ $\left(2 \pi / \gamma_{\mathrm{p}}\right)^{2}\left\langle(T-\langle T\rangle)^{2} /\langle T\rangle^{4}\right.$ in MPM (2):

$$
\sigma_{B}^{\mathrm{pm}}=\sigma_{B}^{0} \eta_{\mathrm{pm}},
$$

where

$$
\sigma_{B}^{0}=\frac{\sigma \sqrt{2}}{\gamma_{\mathrm{p}} t_{N} \sqrt{S_{0}^{2}-\Lambda^{2}}}=\frac{1}{\gamma_{\mathrm{p}} t_{N} K} \sqrt{\frac{W\left(\omega_{r}\right) \omega_{r} Q}{A_{0}^{2}-(\Lambda / K)^{2}}} .
$$

For small $\Lambda$, the correction factor $\eta_{\mathrm{pm}}$ in relation to the signal relaxation reads

$$
\eta_{\mathrm{pm}}^{2} \approx \mathrm{e}^{\xi} \cosh \xi, \quad \xi=t_{N} / T_{2}
$$

Similar computation, using (11) for the LSM case (3), produces

$$
\begin{gathered}
\sigma_{B}^{\mathrm{ls}}=\sigma_{B}^{0} \eta_{\mathrm{ls}} f_{c} \sqrt{3 n T^{0} / t_{N}}, \\
\eta_{\mathrm{ls}}^{2} \approx 3 \mathrm{e}^{\xi}\left[\left(2+\xi^{2}\right) \sinh \xi-2 \xi \cosh \xi\right] / \xi^{3} .
\end{gathered}
$$

The noise correlation factor $f_{c}$ obtained by substitution of (17) into (12) is defined by the relation

$$
\begin{aligned}
f_{c}^{2} & =\frac{\sinh \beta+\left(\tilde{\omega}_{r} \tau_{c}\right)^{-1} \sin \beta \tau_{c}\left(\tilde{\omega}_{r}-\omega_{0}\right)}{\cosh \beta-\cos \beta \tau_{c}\left(\tilde{\omega}_{r}-\omega_{0}\right)}, \\
\beta & =\frac{n T^{0}}{2 \tau_{c}}=\frac{\pi n}{2 \alpha Q} .
\end{aligned}
$$

With the assumption of an undamped signal and ideal comparator determining zero crossing times, we obtain a simplified expression for MSD in the MPM case

$$
\sigma_{B}^{\mathrm{pm}}=\frac{\sqrt{2 k_{b} T R \omega_{r} / Q}}{\gamma_{\mathrm{p}} t_{N} A_{0}}(1+\phi), \quad \phi=\frac{Q}{K}-1,
$$

where the MSD dependence on the frequency is presented in a correction $\phi$, and $\phi=0$ for $\alpha=1$. The random measurement error for the LSM can be written as

$$
\sigma_{B}^{1 \mathrm{~s}}=\frac{4 \sqrt{3 k_{b} T R / t_{N}}}{\gamma_{\mathrm{p}} t_{N} A_{0}}(1+\lambda)
$$

where the dependence of MSD on the quality factor $Q$ and the frequency is presented in a correction $\lambda$ defined by the relation $(\lambda \rightarrow 0$ for $\alpha=1$ and $Q \rightarrow \infty)$ :

$$
\lambda=\frac{Q f_{c}}{K} \sqrt{\frac{\beta}{2}}-1 \text {. }
$$

Expression (24) is valid only in the case $t_{N} \gg \tau_{c}$; further, we assume that the quality factor satisfies this condition. We do not consider the case $t_{N} \approx \tau_{c}$ as it corresponds to a very large systematic measurement error.

\section{Dependence of the measurement error on the sensor quality factor}

Expression (23) illustrates the known result that the MSD of measurements in MPM is proportional to $Q^{-1 / 2}$ in a range of signal frequencies $\omega_{0}$ close to the resonant circuit frequency $\omega_{r}$. Therefore, the random error decreases for a larger quality 


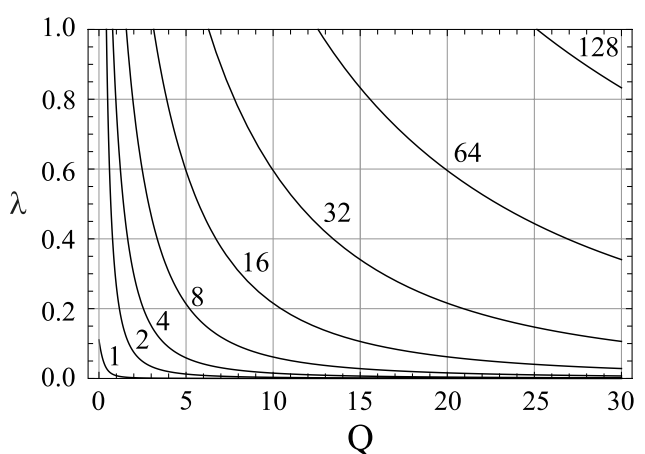

(a)

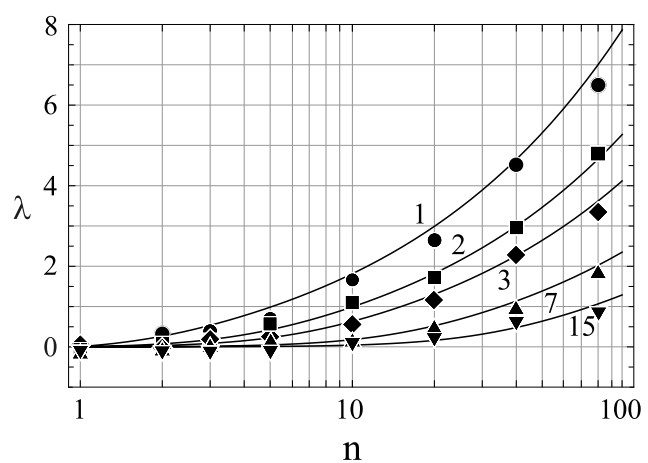

(b)

Figure 1. Dependence of the correction $\lambda$ for the perfect tuning $\omega_{0}=\omega_{r}(a)$ on the quality factor $Q$ (the digitization step $n$ values are shown by numbers) and $(b)$ on the digitization step $n$ (the $Q$ values are shown for each curve).

factor. However, the correlation time increases, leading to systematic error growth and also the actual measurement time decreases due to the natural nuclear magnetization signal relaxation, i.e., signal attenuation. Consider MSD in the LSM (24) at exact tuning of the receiving circuit to the input signal precession frequency $\left(\omega_{0}=\omega_{r}\right)$. Figure $1(a)$ shows the dependence of $\lambda$ on various values of $n$; the MSD dependence on the quality factor for $Q \geqslant 10$ (characteristic for the majority of proton sensors) and the digitization step, $n=1-8$, is not significant $(\lambda \ll 1)$. When $Q \gg \pi n / 2$, the dependence of $\lambda$ on the quality factor can be approximated by

$$
\lambda \cong \frac{n^{2} \pi^{2}-9}{96 Q^{2}} ;
$$

from which it follows that at large $Q$ the correction $\lambda$ tends to zero. However, for the increased digitization step $n T^{0} / 2$, its dependence on the quality factor becomes significant.

A device based on the registration system of a protonprecession magnetometer described above was built to test experimentally the predicted properties. The device included a stable sinusoidal signal generator, a noise generator and an electronic mixer which mixed the signal with noise, amplified the resulting signal and transmitted it into the detector circuit. As the noise component of the signal is much larger than the noise level of the detector circuit, one can neglect the latter. Signal processing was performed by the LSM (3). The quality factor $Q$ value was tuned by the addition of resistors in the electric circuit of the proton sensor. The dependence of MSD on $n$ (or equivalently, on the digitization period) was measured. The results presented in figure $1(b)$ show good correspondence to the predicted dependences.

\section{Dependence of the measurement error on output signal frequency}

Consider the general dependence of the random error on the frequency. The simplest and the best known result is obtained for MPM processing. It follows from (15) that the frequency dependence of MSD actually reproduces an inverse transfer function of the sensor receiving circuit. Correspondingly, the essential frequency dependence of the error of the measured signal is observed at large quality factors.

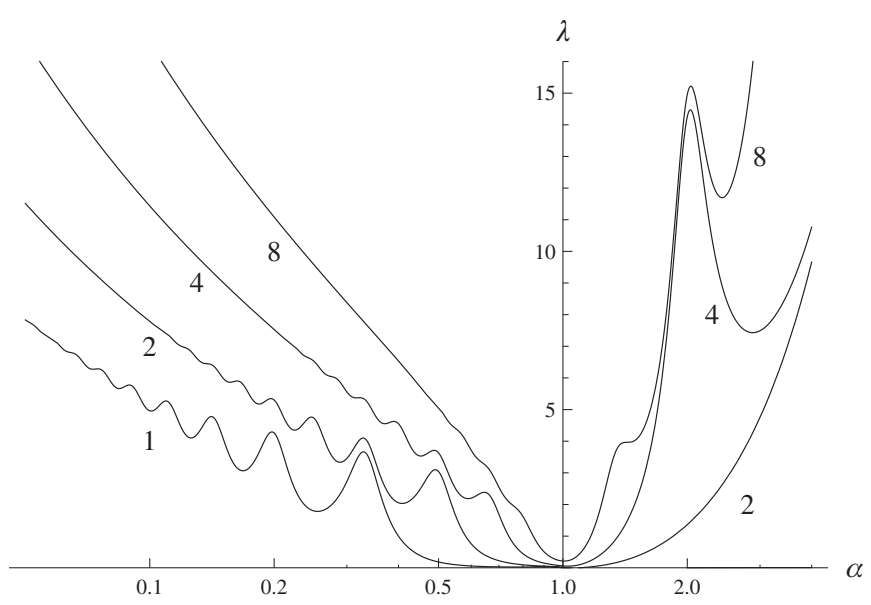

Figure 2. Dependence of the correction $\lambda$ for $Q=5$ on the frequency ratio $\alpha$ for $n=1,2,4,8$.

According to (24), the entire dependence of the measurement MSD in the LSM on the input signal frequency $\omega_{0}$ and resonance frequency of the receiving circuit $\omega_{r}$ is concentrated in the correction factor $\lambda$. An example of $\lambda$ dependence on the frequency ratio $\alpha$ at the quality factor $Q=5$ and various digitization steps is shown in figure 2 . The analysis shows that MSD frequency dependence for linear regression processing considerably differs from the corresponding MPM dependence. For $\omega_{0} \approx \omega_{r}$, there exists a plateau whose width increases with the reduction of the number $n$ of the processed half-periods. One also observes local minima at considerable circuit detuning; their number increases with the digitization step $n$. The observed $\lambda$ dependence on the frequency is related to the modulation of the inverse transfer function by the correlation coefficient $f_{c}$. This coefficient is the periodic function (see figure 3) with minima localized at $\alpha \rightarrow \infty$ and $\alpha \approx n[2 m+(1+\cos \pi n) / 2] /\left[(2 m+(1+\cos \pi n) / 2)^{2}-1\right]$, where $m=1,2, \ldots$. Maxima of this function are at $\alpha \approx n /[2 m-(1-\cos \pi n) / 2]$. It can be easily seen that the positions of the extrema do not depend on the quality factor. The increase of this factor leads to the growth of the amplitude of the correlation coefficient that implies a larger contribution of the correlations at a large deviation of the signal frequency from the circuit frequency. 


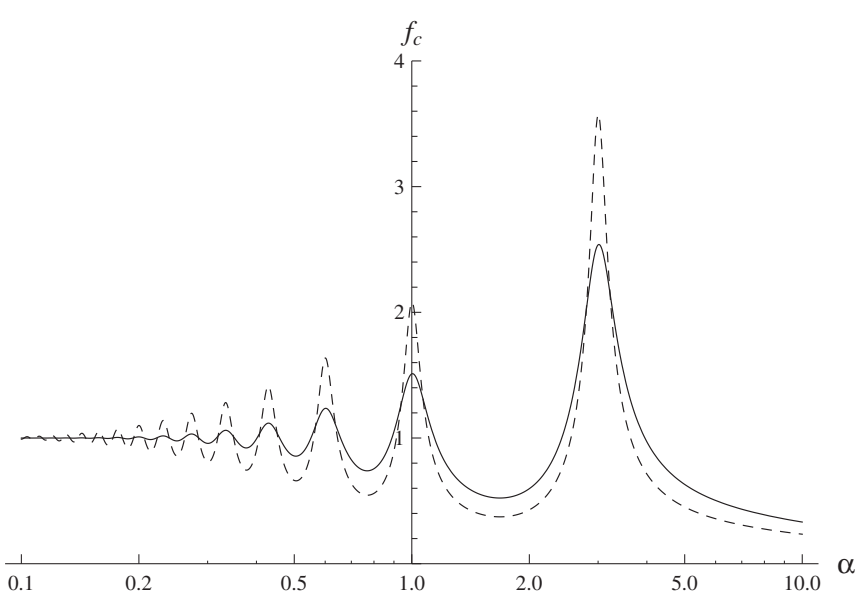

(a)

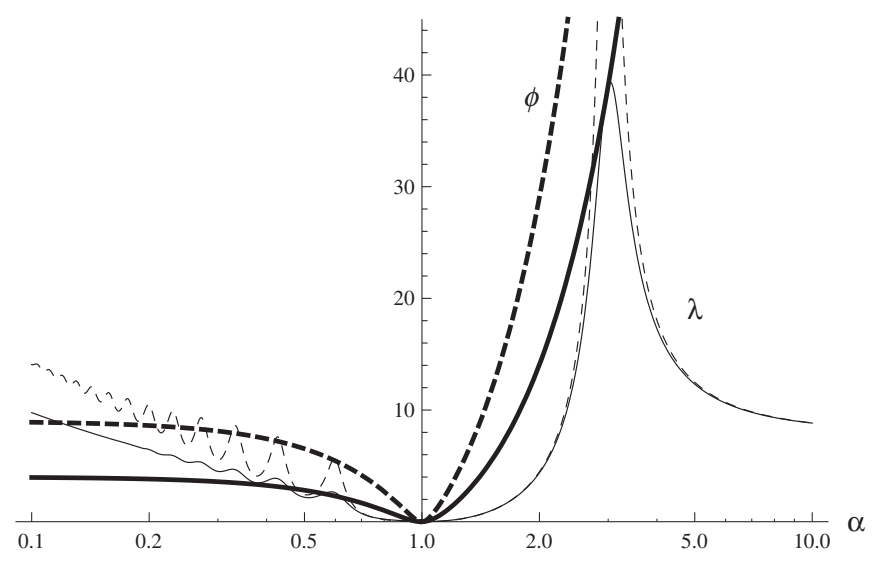

(b)

Figure 3. (a) Dependence of $f_{c}$ on the frequency ratio $\alpha$ for $n=3, Q=5$ (solid) and $Q=10$ (dashed). (b) The comparison of MSD frequency dependences for MPM and LSM. Dependence of the corrections $\lambda$ and $\phi$ (thick curves) on the frequency ratio $\alpha$ for $n=3, Q=5$ (solid) and $Q=10$ (dashed).

Smoothing of MSD frequency dependence in the vicinity of $\omega_{0} \approx \omega_{r}$ (see figure $3(b)$ ) is explained by merging of three minima: the minimum of the inverse transfer function and two local minima of the correlation coefficient at $\alpha \approx$ $(n \pm 1) /(n \pm 2)$. Setting the random error value and using the expressions for $\lambda$, one can estimate a range of accepted frequency values.

Find the frequency estimate in the vicinity of the first local minima $\omega_{r}(n \pm 1) /(n \pm 2)$ as a function of the correction $\lambda$. It is convenient to use expression (14) in the limit of broadband noise. In the case when $Q \geqslant n / 2$, in the sum one can retain only the terms with the closest central frequencies. Then the dependence of $Q$ can be neglected and the expressions for the frequency limit read

$$
\omega_{0}^{ \pm}=\omega_{r} \sqrt{\frac{\lambda(\lambda+2)+1}{(1 \pm 2 / n)^{2} \lambda(\lambda+2)+1}}
$$

where the plus (minus) sign corresponds to the lower (upper) frequency boundary value. This formula leads to the estimate of the frequency range defined by the MSD acceptable level, for example, $\sqrt{2} \sigma_{\min }(\lambda=\sqrt{2}-1)$. The estimate for the bandwidth for $n>1$ is approximated by $\Delta \omega_{0} / \omega_{r} \equiv\left(\omega_{0}^{-}-\right.$ $\left.\omega_{0}^{+}\right) / \omega_{r} \approx 2 / n$, which means that the effective bandwidth $\Delta \omega_{0}$ is larger than the bandwidth $\omega_{r} / Q$ of the receiving circuit, which coincides with the bandwidth for $\sigma_{B}^{\mathrm{pm}}$ at the level $\phi=\sqrt{2}-1$. For convenience, we present the exact results in table 1 where values of the frequency limits, the corresponding bandwidth and effective quality factor $Q_{\text {ef }}$ corresponding to this band (the effective bandwidth of the receiving circuit at the MSD level significantly increases for small $n$ ) are calculated as functions of the digitization step.

For large $Q$ values, the shape of the $\lambda$ curves does not vary significantly with $Q$ change. The dependence of $\lambda$ on the quality factor is expressed only by the proportionality of the maxima value to $Q$. On the other hand, the digitization step distance $n T^{0} / 2$ influences significantly the shape of the $\lambda$ curves.
Table 1. Dependence of the bandwidth at the level $\sqrt{2} \sigma_{\min }$ and the effective quality factor $Q_{\text {ef }}$ on the step $n$ for LSM processing.

\begin{tabular}{rllll}
\hline$n$ & $\omega_{0}^{+} / \omega_{r}$ & $\omega_{0}^{-} / \omega_{r}$ & $\Delta \omega_{0} / \omega_{r}$ & $Q_{\text {ef }}$ \\
\hline 1 & 0.45 & $\infty$ & $\infty$ & 0 \\
2 & 0.65 & 1.55 & 0.90 & 1.11 \\
4 & 0.80 & 1.24 & 0.44 & 2.26 \\
8 & 0.89 & 1.12 & 0.22 & 4.5 \\
16 & 0.95 & 1.06 & 0.11 & 9.0 \\
32 & 0.97 & 1.03 & 0.06 & 18 \\
64 & 0.99 & 1.01 & 0.03 & 37 \\
\hline
\end{tabular}

Registration band widening due to signal regression processing methods similar to the LSM was established experimentally in 1994 within the framework of the studies performed in the Quantum Magnetometry Laboratory (www.magnetometer.ru) concerning the increase of aeromagnetometer operation. This effect was successfully used in the design of new magnetometers. The theoretical description of this effect is presented in this paper.

\section{Discussion}

The presented results show that the application of regression algorithms for small values of digitization step considerably extends the range of measured magnetic fields both without adjustment of the receiving circuit and loss in sensitivity, and also relaxes requirements on the quality factor of the receiving circuit. One can obtain the same registration band in MPM reducing the quality factor down to values of the order of unity, which would essentially increase the measurement error. Only in the case of a considerable drop in the number of signal periods ( $n$ is of the order of several dozens) does the registration band of the magnetometer with MPM become comparable to the corresponding band for regression treatment. 
As we show in section 3, the application of the regression methods in the frequency selective system of a device (not only a magnetometer) may significantly reduce the influence of the frequency response of a frequency selective registration system on the measurement error. The frequency response in this case is determined mainly by the signal processing algorithm and by the harmonic signal envelope. In particular, for a measurement of the signal frequency using the LSM of period measurement one can significantly enlarge the bandwidth for which the device error is minimal. Weak dependence of an error on the frequency response means that measurement error is determined by the ratio of spectral noise density to signal instead of noise/signal ratio. These considerations lead us to the conclusion that application of the regression methods may lead to changes in the architecture and optimization criteria of new electronic equipment.

There is a multitude of methods devoted to the estimation of the harmonic signal frequency ([12-14] and references therein). Discrete-time observation processing usually allows us to obtain the signal parameters even in the case of low signal/noise ratio. But these methods are quite complicated and therefore in some applications their use for fast frequency estimates is not convenient as it requires large computational resources. The signal processing algorithms considered in this paper are useful in devices (variants of the period or frequency meter $[15,16]$ ) where the digitization is based on the zero crossing in the case of a good signal/noise ratio and correlation time is larger or comparable to the signal period. These algorithms are expected to be not complex but fast and precise.

\section{Conclusions}

The choice of the specific processing algorithm is important for measuring device design. Particularly, the application of regression methods for proton magnetometers provides additional resources for the extension of the field range without magnetometer tuning. The implementation of the broadband mode leads to considerable reduction of the hardware part systematic error and simplifies the system of search and tracking of the precession signal frequency and phase. Requirements on the accuracy of synchronous polarization are also relaxed. Additionally, it is possible to increase operation speed without loss in sensitivity due to smaller transient time. The presented conclusions about the regression methods can be observed in other applications; they manifest in the processing of correlated data of the harmonic signals of general type.

\section{Acknowledgments}

The work was partially supported by ISTC (International Scientific Technology Center) grant no 3753.

\section{References}

[1] Primdahl F 1998 Scalar magnetometers for space applications Measurement Techniques in Space Plasmas (Field Geophysical Monograph vol 103) (New York: Wiley) pp 85-99

[2] Ripka P 2001 Magnetic Sensors and Magnetometers (Boston, MA: Artech House) chapter 7

[3] Kernevez F and Glenat H 1991 Description of a high-sensitivity CW scalar DNP-NMR magnetometer IEEE Trans. Magn. 27 5402-4

[4] Denisov A Y, Sapunov V A and Dikusar O V 1999 Calculation of the measurement error of a digital-processor nuclear-precession magnetometer Geomagn. Aeron. 39 68-73 (in Russian)

Denisov A Y, Sapunov V A and Dikusar O V 1999 Geomagn. Aeron. 39737 (abstract only)

[5] Sapunov V A, Denisov A Y, Denisova O V and Saveliev D V 2001 Proton and Overhauser magnetometers metrology Contrib. Geophys. Geod. 31 119-24

[6] Mohr P J, Taylor B N and Newell D B 2012 CODATA recommended values of the fundamental physical constants: 2010 Rev. Mod. Phys. 84 1527-79

[7] Mischie S and Toma L 2006 Extending the frequency measurement of a single sinusoid above the Nyquist limit based on zero crossings method Facta Univ. Ser.: Electron. Energ. 19 1-11

[8] Farrell E J and Grosch C B 1965 Determination of period from times of zeros Proc. IEEE 53 2162-3

[9] Hancke G P 1990 The optimal frequency estimation of a noisy sinusoidal signal IEEE Trans. Instrum. Meas. 39 843-6

[10] Chibane Y, Lamoreaux S K, Pendlebury J M and Smith K F 1995 Minimum variance of frequency estimations for a sinusoidal signal with low noise Meas. Sci. Technol. 6 1671-8

[11] Nyquist H 1928 Thermal agitation of electric charges in conductors Phys. Rev. 32 110-7

[12] Trittler S 1985 Estimating the frequency of a noisy sinusoid by linear regression IEEE Trans. Inform. Theory 31 832-5

[13] Trittler S and Hamprecht F A Near optimum sampling design and an efficient algorithm for single tone frequency estimation J. Digit. Signal Process. 19 628-39

[14] Park S Y, Song Y S, Kim H J and Park J 2011 Improved method for frequency estimation of sampled sinusoidal signals without iteration IEEE Trans. Instrum. Meas. 60 2828-34

[15] Korolev I A, Alekseenko N N, Porodnov B T, Sapunov V A and Savel'ev D V 2003 A digital deformation vacuum gauge with an LC-generator Meas. Tech. 46 865-71

[16] Sheng D, Li S, Dural N and Romalis M V 2013 Subfemtotesla scalar atomic magnetometry using multipass cells Phys. Rev. Lett. 110160802 